\title{
Tractability and learnability arising from algebras with few subpowers
}

\author{
Paweł Idziak \\ Jagiellonian University \\ Theoretical Computer Science Department \\ Kraków, Poland \\ idziak@tcs.uj.edu.pl \\ Ralph McKenzie \\ Vanderbilt University \\ Department of Mathematics \\ Nashville, Tennessee USA \\ ralph.n.mckenzie@vanderbilt.edu
}

\author{
Petar Marković \\ Department of Mathematics and Informatics \\ University of Novi Sad \\ Novi Sad, Serbia \\ pera@im.ns.ac.yu \\ Matthew Valeriote \\ Department of Mathematics and Statistics \\ McMaster University \\ Hamilton, Ontario \\ Canada L8S 4K1 \\ matt@math.mcmaster.ca
}

\author{
Ross Willard \\ Department of Pure Mathematics \\ University of Waterloo \\ Waterloo, Ontario \\ Canada \\ rdwillareuwaterloo.ca
}

\begin{abstract}
A $k$-edge operation $\varphi$ on a finite set $A$ is a $k+1$-ary operation that satisfies the identities

$$
\begin{aligned}
\varphi(x, x, y, \ldots, y) & \approx \varphi(x, y, x, y, \ldots, y) \approx y \\
\varphi(y, y, y, x, y, \ldots, y) & \approx \varphi(y, y, y, y, x, y, \ldots, y) \approx \cdots \\
\cdots & \approx \varphi(y, y, y, \ldots, y, x) \approx y .
\end{aligned}
$$
\end{abstract}

We prove that any constraint language $\Gamma$ that, for some $k>1$, has a k-edge operation as a polymorphism is globally tractable. We also show that the set of relations definable over $\Gamma$ using quantified generalized formulas is polynomially exactly learnable using improper equivalence queries.

Special instances of k-edge operations are Mal'cev and near-unanimity operations and so this class of constraint languages includes many well known examples.

\section{Introduction}

A wide variety of combinatorial problems can be expressed within the framework of the Constraint Satisfaction
Problem (CSP). Given an instance of the CSP the aim is to determine if there is an assignment of values to the variables of the instance that satisfies all of its constraints. While the class of all CSPs forms an NP-complete class of problems there are many naturally defined subclasses that are tractable (i.e., lie in the class $\mathbf{P}$ ). A main research goal is to identify the subclasses of the CSP that are tractable.

One common way to define a subclass of the CSP is to restrict the relations that appear in the constraints of an instance to a specified set of relations over some fixed domain, called a constraint language. The Dichotomy Conjecture of Feder and Vardi [14] states that for any constraint language $\Gamma$, the corresponding subclass of the CSP, denoted $\operatorname{CSP}(\Gamma)$, is either NP-complete or tractable. The Dichotomy Conjecture has been verified in a number of special cases, most notably over domains of size 2 [20] and size 3 [4]. Over larger domains, a number of general results have been obtained [7, 3, 12].

In this paper we introduce a condition that when satisfied by a constraint language $\Gamma$, guarantees that the subclass $\operatorname{CSP}(\Gamma)$ is tractable. The polynomial-time algorithm that we use to establish tractability is essentially that presented by Dalmau in [12] and our result generalizes his and also generalizes earlier results of Feder and Vardi, Bulatov, 
and Jeavons, Cohen and Cooper [14, 6, 17]. The class of constraint languages that satisfy our condition is quite large and includes some well known constraint languages such as those that have Mal'cev or near unanimity polymorphisms.

A polymorphism of a set of relations $\Gamma$ over some set $A$ is a finitary operation on $A$ that preserves all of the relations in $\Gamma$. Work of Jeavons and his co-authors has shown that the tractability of a constraint language is determined by the set of its polymorphisms and many tractability results can be expressed in terms of the existence of certain kinds of polymorphisms [8]. A benefit of focusing on polymorphisms is that it allows the introduction of ideas and techniques from universal algebra into the study of tractability and in this paper we adopt this algebraic approach.

Our main result is that if a constraint language $\Gamma$ has a $k$ edge operation (see Definition 3.2) as a polymorphism then Dalmau's algorithm can be used to solve in polynomial time all instances in $\operatorname{CSP}(\Gamma)$. This algorithm can be regarded as a natural generalization of the familiar Gaussian elimination algorithm for solving systems of linear equations since it makes essential use of the fact that, in this case, all solution sets of instances of $\operatorname{CSP}(\Gamma)$ have small generating sets (akin to bases of vector spaces) when considered as universes of particular algebras.

On the other hand, we argue that if a constraint language $\Gamma$ fails to have a $k$-edge operation as a polymorphism then no "Gaussian-like" algorithm can solve all instances of $\operatorname{CSP}(\Gamma)$ in polynomial-time. So, in some sense, our result sharply determines the scope of algorithms like Dalmau's to quickly settle instances of the CSP.

In Section 5 we establish that if $\Gamma$ is a set of relations over a finite set that is invariant under a $k$-edge operation then the set of relations defined by quantified generalized formulas over $\Gamma$ is exactly learnable, in polynomial time, via an algorithm that makes improper equivalence queries. This extends the result of Bulatov, Chen, and Dalmau in [5] and earlier results of Dalmau and Jeavons [13].

\section{Preliminaries}

Definition 2.1 An instance of the constraint satisfaction problem is a triple $P=(V, A, \mathcal{C})$ with

- $V$ a non-empty, finite set of variables,

- A a non-empty, finite set (or domain),

- $\mathcal{C}$ a set of constraints $\left\{C_{1}, \ldots, C_{q}\right\}$ where each $C_{i}$ is a pair $\left(\vec{s}_{i}, R_{i}\right)$ with

- $\vec{s}_{i}$ a tuple of variables of length $m_{i}$, called the scope of $C_{i}$, and

- $R_{i}$ an $m_{i}$-ary relation over $A$, called the constraint relation of $C_{i}$.
Given an instance $P$ of the CSP we wish to answer the following question:

Is there a solution to $P$, i.e., is there a function $f: V \rightarrow A$ such that for each $i \leq q$, the $m_{i}$-tuple $f\left(\vec{s}_{i}\right) \in R_{i}$ ?

In general, the class of CSPs is NP-complete (see [17]), but by restricting the nature of the constraint relations that are allowed to appear in an instance of the CSP, it is possible to find natural subclasses of the CSP that are tractable.

Definition 2.2 Let $A$ be a domain and $\Gamma$ a set of finitary relations over $A . \operatorname{CSP}(\Gamma)$ denotes the collection of all instances of the CSP with domain $A$ and with constraint relations coming from $\Gamma . \Gamma$ is called the constraint language of the class $\operatorname{CSP}(\Gamma)$.

Definition 2.3 Call a finite constraint language $\Gamma$ tractable if the class of problems $\operatorname{CSP}(\Gamma)$ is tractable (i.e., lies in $\mathbf{P}$ ). If $\Gamma$ is infinite and each finite subset $\Gamma^{\prime}$ of $\Gamma$ is tractable then we say that $\Gamma$ is tractable. If $\Gamma$ is infinite and the entire class $\operatorname{CSP}(\Gamma)$ is in $\mathbf{P}$ then we say that $\Gamma$ is globally tractable.

$\Gamma$ is said to be NP-complete if for some finite subset $\Gamma^{\prime}$ of $\Gamma$, the class of problems $\operatorname{CSP}\left(\Gamma^{\prime}\right)$ is $\mathbf{N P}$-complete.

A key problem in this area is to classify the (globally) tractable constraint languages. Note that in this paper we will assume that $\mathbf{P} \neq \mathbf{N P}$. Feder and Vardi [14] conjecture that every finite constraint language is either tractable or is NP-complete.

The natural duality between sets of relations (constraint languages) over a set $A$ and sets of operations (algebras) on $A$ has been studied by algebraists for some time. Jeavons and his co-authors [16] have shown how this link between constraint languages and algebras can be used to transfer questions about tractability into equivalent questions about algebras. In the remainder of this section we present a concise overview of this connection.

Definition 2.4 Let $A$ be a non-empty set.

1. Let $R$ be an $n$-ary relation over $A$ and $f(\bar{x})$ an $m$ ary function over $A$ for some $n, m \geq 0$. We say that $R$ is invariant under $f$ and that $f$ is a polymorphism of $R$ if for all $\vec{a}_{i} \in R$, for $1 \leq i \leq m$, the $n$-tuple $f\left(\vec{a}_{1}, \ldots, \vec{a}_{m}\right) \in R$ whose $i$-th coordinate is equal to $f\left(\vec{a}_{1}(i), \ldots, \vec{a}_{m}(i)\right)$.

2. For $\Gamma$ a set of relations over $A, \operatorname{Pol}(\Gamma)$ denotes the set of functions on A that are polymorphisms of all the relations in $\Gamma$.

3. For $F$ a set of finitary operations on $A, \operatorname{Inv}(F)$ denotes the set of all finitary relations on $A$ that are invariant under all operations in $F$. 
4. For $\Gamma$ a constraint language over $A,\langle\Gamma\rangle$ denotes $\operatorname{Inv}(\operatorname{Pol}(\Gamma))$, the relational clone on $A$ generated by $\Gamma$.

We note that given a set of relations $\Gamma$ over a finite set $A$, the relational clone generated by $\Gamma$ is equal to the set of relations over $A$ definable via $\Gamma$ using primitive-positive formulas (or conjunctive queries) (see [16]).

Theorem 2.5 ([16]) Let $\Gamma$ be a constraint language on a finite set. If $\Gamma$ is tractable then so is $\langle\Gamma\rangle$. If $\langle\Gamma\rangle$ is NPcomplete then so is $\Gamma$.

We refer the reader to [9] or [19] for the basics of universal algebra, in particular to the notions of a cartesian power and a subuniverse of an algebra. By an algebra we mean the following:

Definition 2.6 An algebra $\mathbf{A}$ is a pair $(A, F)$ where $A$ is a non-empty set and $F$ is a (possibly infinite) collection of finitary operations on $A$. The operations in $F$ are called the basic operations of $\mathbf{A}$. A term operation of an algebra $\mathbf{A}$ is a finitary operation on $A$ that can be obtained by repeated compositions of the basic operations of $\mathbf{A}$.

Definition 2.7 Let $\mathbf{A}=(A, F)$ be a finite algebra and $\Gamma$ a constraint relation over $A$.

1. $\mathbf{A}_{\Gamma}$ denotes the algebra $(A, \mathrm{Pol}(\Gamma))$ and $\Gamma_{\mathbf{A}}$ denotes the constraint language $\operatorname{Inv}(F)$

2. We call the algebra A tractable, globally tractable, or $\mathbf{N P}$-complete if the constraint language $\Gamma_{\mathbf{A}}$ is.

Note that if $\mathbf{A}$ is an algebra, then $\operatorname{Inv}(\mathbf{A})$ coincides with the set of all subuniverses of finite cartesian powers of $\mathbf{A}$.

In algebraic terms, Theorem 2.5 states that a constraint language $\Gamma$ is tractable (or NP-complete) if and only if the algebra $\mathbf{A}_{\Gamma}$ is. So, the problem of characterizing the tractable constraint languages can be reduced to the problem of characterizing the tractable finite algebras.

\section{Algebras with few subpowers}

Given a finite algebra $\mathbf{A}$ we can associate to it a function that counts the number of subalgebras of finite powers of A. The main results of this paper rely on the companion paper [2], wherein this function is extensively studied. Earlier work by Dalmau and Jeavons [11, 13] and Chen [10] deal with notions closely related to this function.

Definition 3.1 Let $\mathbf{A}$ be a finite algebra.

1. For $n$ a positive integer, define $s_{\mathbf{A}}(n)$ to be the logarithm, base 2, of the cardinality of the set of all subuniverses of the algebra $\mathbf{A}^{n}$.
2. We say that $\mathbf{A}$ has few subpowers if the function $s_{\mathbf{A}}$ can be bounded by some polynomial.

We note that in [10] algebras (or sets of operations over a finite set) with few subpowers are said to be polynomially expressive.

In this paper we rely on the following definition and result from [2] that characterize finite algebras with few subpowers.

Definition 3.2 A $k$-edge operation on a set $A$ is a $k+1$-ary operation $e(\bar{x})$ on A that satisfies the equations

$$
\begin{aligned}
e(x, x, y, y, y, \ldots, y, y) & \approx y \\
e(x, y, x, y, y, \ldots, y, y) & \approx y \\
e(y, y, y, x, y, \ldots, y, y) & \approx y \\
e(y, y, y, y, x, \ldots, y, y) & \approx y \\
& \vdots \\
e(y, y, y, y, y, \ldots, x, y) & \approx y \\
e(y, y, y, y, y, \ldots, y, x) & \approx y
\end{aligned}
$$

A term e of an algebra $\mathbf{A}$ is a k-edge term for $\mathbf{A}$ if $e^{\mathbf{A}}$ is a $k$-edge operation on A.

Examples 3.3 Let $A$ be a set.

1. A Mal'cev operation on $A$ is a ternary operation $p(x, y, z)$ that satisfies the equations $p(y, x, x) \approx$ $p(x, x, y) \approx y$. It is clear that a ternary operation $p(x, y, z)$ on $A$ is Mal'cev if and only if $p(y, x, z)$ is a 2-edge operation on $A$.

2. A $k$-ary near unanimity operation on $A$ is a $k$-ary operation $t(\bar{x})$ that satisfies the equations

$$
\begin{aligned}
t(x, y, \ldots, y) & \approx t(y, x, y, \ldots, y) \approx \cdots \\
\cdots & \approx t(y, y, \ldots, x) \approx y .
\end{aligned}
$$

If $t$ is a $k$-ary near unanimity operation then the $k+1$ ary operation $e(\bar{x})=t\left(x_{2}, x_{3}, \ldots, x_{k+1}\right)$ is a $k$-edge operation.

3. A $k$-ary operation $g$ on $A$ is a generalized majorityminority (gmm) operation $([5,12])$ if for all $a, b \in A$, we have that either for all $x, y \in\{a, b\}$,

$$
\begin{aligned}
g(x, y, \ldots, y) & =g(y, x, y, \ldots, y)=\cdots \\
\cdots & =g(y, y, \ldots, x)=y
\end{aligned}
$$

or for all $x, y \in\{a, b\}$,

$$
g(y, x, \ldots, x)=g(x, x, \ldots, x, y)=y .
$$

From [2] we know that any algebra with a $k$-ary gmm term operation also has a $k$-edge term operation. 
Theorem 3.4 ([2]) Let A be a finite algebra. Then A has few subpowers if and only if for some $k>0$, A has a $k$-edge term. In this case, $s_{\mathbf{A}}(n)$ is bounded above by a polynomial of degree $k$.

We note that in [2] it is established that if a finite algebra $\mathbf{A}$ fails to have few subpowers then the function $s_{\mathbf{A}}$ is bounded below by an exponential function. This extends the result of Chen for two element algebras found in [10].

One direction of the proof of Theorem 3.4 establishes that in the presence of a $k$-edge term, all subalgebras of finite powers of a finite algebra $\mathbf{A}$ have rather small generating sets. The proof utilizes three auxiliary terms that are derivable from a $k$-edge term.

Lemma 3.5 ([2]) Let A be a finite algebra with a k-edge term e. Then $\mathbf{A}$ also has terms $d(x, y), p(x, y, z)$ and $s\left(x_{1}, x_{2}, \ldots, x_{k}\right)$ satisfying

$$
\begin{aligned}
p(x, y, y) & \approx x \\
p(x, x, y) & \approx d(x, y) \\
d(x, d(x, y)) & \approx d(x, y) \\
s(x, y, y, y, \ldots, y, y) & \approx d(y, x) \\
s(y, x, y, y, \ldots, y, y) & \approx y \\
s(y, y, x, y, \ldots, y, y) & \approx y \\
s(y, y, y, y, \ldots, y, x) & \vdots y .
\end{aligned}
$$

The following two definitions are extensions of those given in $[5,12]$ in the context of generalized majorityminority operations.

Definition 3.6 Suppose $\mathbf{A}$ is an algebra with $k$-edge term e and terms $d, p$, $s$ as in Lemma 3.5. A pair $(a, b) \in A^{2}$ is a minority pair if $d(a, b)=b$.

By an index (of rank $n$ ) we mean a triple $I=(i, a, b)$ where $1 \leq i \leq n$ and $a, b \in A$. It is said to be a minority index if $(a, b)$ is a minority pair. If $S \subseteq A^{n}$, we say that $I$ is witnessed in $S$ if there exist $f, g \in S$ satisfying

- $f(j)=g(j)$ for all $j<i$.

- $f(i)=a$ and $g(i)=b$.

In this case we call $f, g$ witnesses to $I$.

Definition 3.7 Let $\mathbf{A}$ be an algebra with k-edge term e and terms $d, p, s$ as in Lemma 3.5 and suppose $R \subseteq B \subseteq A^{n}$ for some $n>0$.

1. The signature of $B$, denoted by $\mathrm{Sig}_{B}$, is the set of all minority indices of rank $n$ that are witnessed in $B$.

2. We say that $R$ is a representation of $B$ if
- For all $T \subseteq[n]$ with $|T|<k, \operatorname{proj}_{T}(B)=$ $\operatorname{proj}_{T}(R)$. ([n] denotes the set $\left.\{1,2, \ldots, n\}\right)$

- $\operatorname{Sig}_{R}=\operatorname{Sig}_{B}$.

3. $\langle R\rangle_{e}$ denotes the closure of $R$ under the operation $e$ (or equivalently the smallest $n$-ary relation on $A$ that contains $R$ and is invariant under $e$.)

Lemma 3.8 Let $\mathbf{A}$ be a finite algebra with k-edge term e and terms $d, p$, s as in Lemma 3.5. Suppose $\mathbf{B}$ is a subalgebra of $\mathbf{A}^{n}, R$ is a representation of $\mathbf{B}$, and $I=(i, a, b)$ is a minority index witnessed in $B$. Then for all $f \in\langle R\rangle_{e}$ with $f(i)=a$, there exists $g \in\langle R\rangle_{e}$ such that $f, g$ witness $I$.

Proof: Because $I$ is minority and witnessed in $B$, we can choose $f^{*}, g^{*} \in R$ witnessing $I$. Define

$$
g=p\left(f, f^{*}, g^{*}\right)
$$

Because $p(x, y, y) \approx x$ we have $f(j)=g(j)$ for all $j<i$. At coordinate $i$,

$$
g(i)=p(a, a, b)=d(a, b)=b
$$

where the last equality holds because $(a, b)$ is minority.

The proof of the following theorem may be found in [2].

Theorem 3.9 Suppose $\mathbf{A}$ is a finite algebra with $k$-edge term e and terms $d, p, s$ as in Lemma 3.5. If $\mathbf{B}$ is a subalgebra of $\mathbf{A}^{n}$ and $R$ is a representation of $\mathbf{B}$, then $\langle R\rangle_{e}=B$.

As observed in [5] and [12], every subset $B$ of $A^{n}$ has a representation whose size is bounded above by a polynomial in $n$. In fact, if we set $m=\min (k-1, n)$ then $B$ has a representation of size at most $2\left|\operatorname{Sig}_{B}\right|+$ $\sum_{T \subseteq[n],|T|=m}\left|\operatorname{proj}_{T}(B)\right|$. We will call a representation with this property a compact representation of $B$. A straightforward computation (noted in Lemma 4 of [5]) establishes that every subset of $A^{n}$ has a compact representation of size bounded above by a $(k-1)$-degree polynomial in $n$.

Following [11], let us say that an algebra $\mathbf{A}$ is polynomially generated (of degree $m$ ) if every subuniverse of $\mathbf{A}^{n}$ has a generating set whose size is bounded above by a polynomial in $n$ of degree at most $m$. We conclude that if $\mathbf{A}$ has a $k$-edge term then it is polynomially generated of degree $k-1$. The converse to this statement is proved in [2].

Note that given a subset $R$ of $A^{n}$, $R$ will be a compact representation of some subset of $A^{n}$ if and only if it is a compact representation of itself. This can be determined by comparing the size of $R$ with $2\left|\operatorname{Sig}_{R}\right|+$ $\sum_{T \subseteq[n],|T|=m}\left|\operatorname{proj}_{T}(R)\right|$, where $m=\min (k-1, n)$, a calculation that can be carried out in time bounded by a polynomial in $n$ and the size of $R$. 


\section{Tractability}

In this section we argue that, with slight modifications, the polynomial-time algorithm presented in [12] for algebras having a gmm term works for finite algebras having few subpowers. Thus we claim the following result:

Theorem 4.1 Let $\mathbf{A}$ be a finite algebra with few subpowers. The constraint language $\Gamma_{\mathbf{A}}$ consisting of all subuniverses of finite cartesian powers of $\mathbf{A}$ is globally tractable.

Corollary 4.2 Any constraint language over a finite set $A$ that has, for some $k>1$, a k-edge operation as a polymorphism is globally tractable.

Note that this settles a conjecture posed by Chen in [10] and Conjecture 1 from Dalmau's thesis [11], namely that any algebra that is polynomially generated is tractable.

For the remainder of this section, we assume that $\mathbf{A}$ is a finite algebra of the form $\left\langle A, \varphi\left(x_{1}, \ldots, x_{k+1}\right)\right\rangle$, where $\varphi$ is a $k$-edge operation. Note that in order to prove Theorem 4.1 it will suffice to consider algebras of this form.

For $P=\left(\left\{v_{1}, \ldots, v_{n}\right\}, A,\left\{C_{1}, \ldots, C_{m}\right\}\right)$ an instance of $\operatorname{CSP}\left(\Gamma_{\mathbf{A}}\right)$, and for $0 \leq l \leq m$, let $P_{l}=\left(\left\{v_{1}, \ldots, v_{n}\right\}, A,\left\{C_{1}, \ldots, C_{l}\right\}\right)$, be the instance of $\operatorname{CSP}\left(\Gamma_{\mathbf{A}}\right)$ obtained from $P$ by only using the first $l$ constraints of $P$ and let $R_{l}$ denote the set of solutions of $P_{l}$.

In essence, the Dalmau Algorithm starts off with a compact representation of $R_{0}\left(=A^{n}\right)$ and then recursively constructs compact representations for $R_{l}$, for $0<l \leq m$. At the end of this recursion, we have $R_{m}$, a compact representation for the set of solutions of $P$, and so $P$ will have a solution if and only if $R_{m}$ is non-empty. Of course, the algorithm can be easily modified so that it outputs a solution of $P$ if one exists.

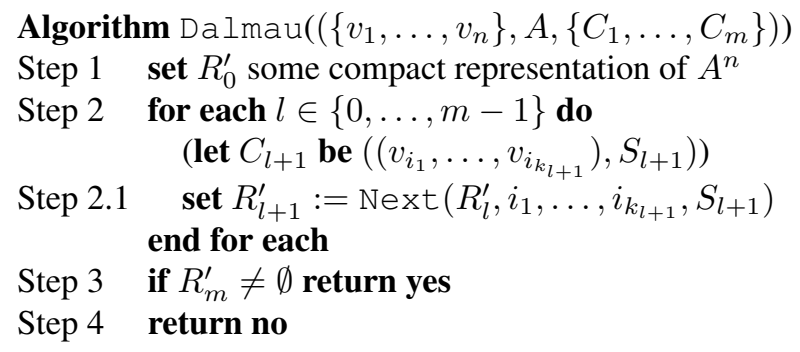

The Dalmau algorithm makes use of a number of procedures that are defined and analyzed in [12]. We now present a description of these procedures, pointing out any necessary modifications, along with estimates of their run-times. Unless otherwise noted, justifications for the correctness and bounds on run-times can be found in Section 4 of [12].
- Nonempty $\left(R^{\prime}, i_{1}, \ldots, i_{j}, S\right)$ receives as input a compact representation $R^{\prime}$ of a subuniverse $R$ of $\mathbf{A}^{n}$ for some $n$, a sequence of elements $i_{1}, \ldots, i_{j}$ from $[n]$, and a subuniverse $S$ of $\mathbf{A}^{j}$. If there is some element $\mathbf{t} \in R$ such that $\operatorname{proj}_{\left\{i_{1}, \ldots, i_{j}\right\}}(\mathbf{t}) \in S$ then Nonempty outputs some member of $R$ with this property and if not, returns the answer "no".

The running time of Nonempty can be bounded by $O\left(\left((n|A|)^{k}+\left|\operatorname{proj}_{\left\{i_{1}, \ldots, i_{j}\right\}}(R)\right|\right)^{k+2}|S| n\right)$.

- Fix-values $\left(R^{\prime}, a_{1}, \ldots, a_{m}\right)$ receives as input a compact representation $R^{\prime}$ of a subuniverse $R$ of $\mathbf{A}^{n}$ for some $n$ and a sequence of elements $a_{1}, \ldots, a_{m}$ from $A$ and outputs a compact representation of the subuniverse

$$
\left\{\mathbf{t} \in R: \operatorname{proj}_{[m]}(\mathbf{t})=\left(a_{1}, a_{2}, \ldots, a_{m}\right)\right\} .
$$

The only change in the definition of Fix-values found in Section 4.2 of [12] is that Step 2.2.1.2 has been removed and Step 2.2.1.1 changed to

$$
\text { set } \mathbf{t}_{5}:=p\left(\mathbf{t}_{1}, \mathbf{t}_{2}, \mathbf{t}_{3}\right)
$$

where $p(x, y, z)$ is the term derived from the $k$-edge operation $\varphi$ using Lemma 3.5. See Figure 1 for a presentation of the modified Fix-values. The proof of the correctness of this modified version of Fix-values is similar to the proof found in Section 4.2 of [12] but makes use of our Lemma 3.8.

The running time of $\mathrm{Fix}-\mathrm{values}$ can be bounded by $O\left((n|A|)^{(k+1)(k+2)}\right)$.

- $\operatorname{Next}\left(R^{\prime}, i_{1}, \ldots, i_{j}, S\right)$ receives as input a compact representation $R^{\prime}$ of a subuniverse $R$ of $\mathbf{A}^{n}$ for some $n$, a sequence of elements $i_{1}, \ldots, i_{j}$ from $[n]$, and a subuniverse $S$ of $\mathbf{A}^{j}$. It outputs a compact representation of the subuniverse

$$
R^{*}=\left\{\mathbf{t} \in R: \operatorname{proj}_{\left\{i_{1}, \ldots, i_{j}\right\}}(\mathbf{t}) \in S\right\} .
$$

Note that Next makes use of a similar procedure, called Next-beta, but which has a potentially worse running time.

As noted in Section 4.3 of [12], the running time of each call to Next in line 2.1 of the Da lmau Algorithm is bounded by $O\left((n|A||S|)^{(k+2)^{2}}\right)$.

Corollary 4.3 The algorithm Dalmau correctly decides if an instance $P$ of $\operatorname{CSP}\left(\Gamma_{\mathbf{A}}\right)$ has a solution in time $O\left(m\left(n|A|\left|S^{*}\right|\right)^{(k+2)^{2}}\right)$, where $n$ is the number of variables of $P, m$ is the number of constraints of $P$, and $S^{*}$ is the largest constraint relation occurring in $P$. 


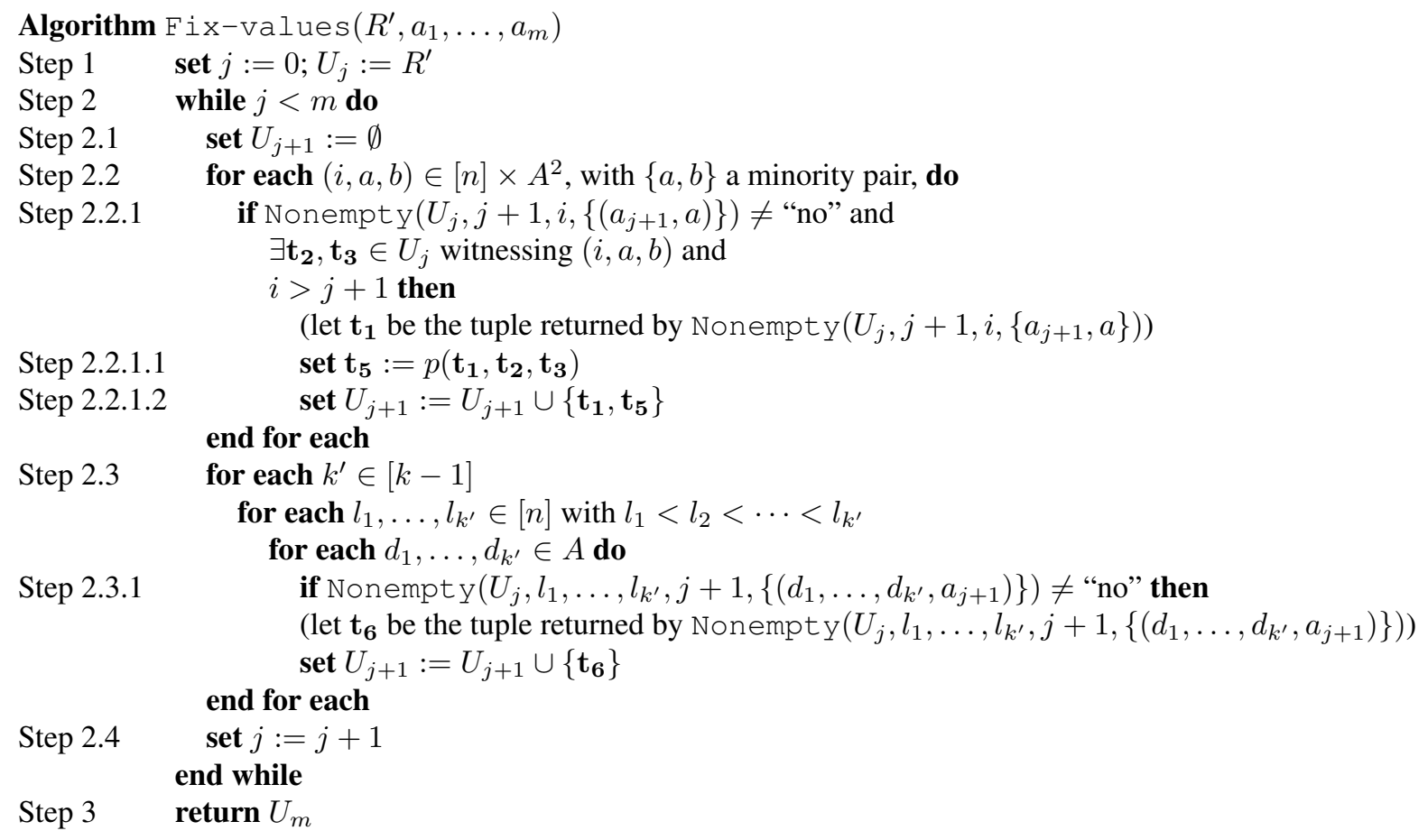

Figure 1. The Fix-values Algorithm

To conclude this section, we note that if $\mathbf{A}$ is a finite algebra that fails to have few subpowers then it is still possible for $\Gamma_{\mathbf{A}}$ to be a tractable constraint language, but even so, no algorithm based on some notion of a "small" generating set for finite subpowers of $\mathbf{A}$ can correctly solve all instances of $\operatorname{CSP}\left(\Gamma_{\mathbf{A}}\right)$ in polynomial time. This is because, as noted earlier, in [2] it is shown that if $\mathbf{A}$ fails to have few subpowers then it is not polynomially generated. In fact, if $\mathbf{A}$ fails to have few subpowers then the function $g_{\mathbf{A}}(n)$ defined to be the least integer $k$ such that each subuniverse of $\mathbf{A}^{n}$ has an at most $k$-element generating set is bounded below by an exponential function.

\section{Learnability}

In this section we show how to modify the proof of Bulatov, Chen, and Dalmau found in [5] to show that when $f$ is a $k$-edge operation on a finite set $A$, there is an algorithm that exactly learns, in polynomial time, the set $\operatorname{Inv}(f)$, encoded by compact representations.

First we give a brief overview of the learning model that is used. More details may be found in Angluin and Kharitonov [1], or in [13,5]. We fix a finite set $A$ and define $X$ to be the set of all finitary tuples over $A$. A concept $c$ is simply a subset of $X$ along with some sort of encoding of it, while a concept class is just a set of concepts.
A learning algorithm for a concept class $C$ is a procedure that, by making specific kinds of queries to an oracle, eventually produces an encoding of a given target concept $t$ from $C$. The model that we adopt is called the exact model with equivalence queries. Learning algorithms in this model are allowed to provide the oracle with a hypothetical encoding $h$ of the target concept $t$ and the oracle either confirms that $h$ indeed encodes $t$ or it returns a counterexample from the symmetric difference of $t$ and the concept coded by $h$. If the hypothesis $h$ codes a concept that does not belong to the class $C$ then the query made to the oracle is said to be improper.

A learning algorithm is said to learn a concept class $C$ if for every target concept $t$ from $C$, it halts with an encoding of $t$. The algorithm runs in polynomial time if its run-time can be bounded by a polynomial in the size of the encoding of the target concepts and the largest counterexample returned by the oracle. A concept class $C$ is polynomially learnable with equivalence queries if there is a learning algorithm that learns $C$ and that runs in polynomial time. If there is a polynomial time learning algorithm that only makes proper equivalence queries to the oracle then $C$ is said to be polynomially learnable with proper equivalence queries; otherwise, $C$ is said to be polynomially learnable with improper equivalence queries.

A related notion is that of being polynomially evaluable 
([13]). A concept class $C$ has this property if there is a polynomial time algorithm that, on input the code of a concept $c$ from $C$ and a tuple $\mathbf{x}$ from $X$, determines if $\mathbf{x}$ is in $c$ or not.

For the remainder of this section, let $A$ be a finite set, $f\left(x_{1}, \ldots, x_{k+1}\right)$ a $k$-edge operation on $A$, and $d, p$, and $s$ the terms derived from $f$ as in Lemma 3.5. We let $\operatorname{Inv}(f)$ denote the set of all finitary relations over $A$ that are invariant under $f$. As in Definition 3.7, for $R \subseteq A^{n},\langle R\rangle_{f}$ denotes the smallest relation invariant under $f$ that contains $R$ (or equivalently, the subuniverse of $(A, f)^{n}$ generated by $R)$.

For $\operatorname{Inv}(f)$ to be considered as a concept class, we need to specify an encoding for each of its members and for convenience, rather than using the notion of a signature developed in Section 4 of [5], we will use compact representations. In what follows, we could just as easily adopt the signature formalism from [5].

Recall that every $n$-ary relation $R$ over $A$ has a compact representation (relative to $f, d, p$ and $s$ ) and that if $R$ is invariant under $f$ then every one of its compact representations generates $R$ as a subuniverse of $(A, f)^{n}$. Thus, we may use compact representations to code members of $\operatorname{Inv}(f)$.

An example presented in Section 4 of [5] shows that not all compact representations of relations over $A$ are necessarily compact representations of relations invariant under $f$. This necessitates expanding our concept class to include arbitrary relations over $A$, encoded by compact representations via a variation of the $\mathrm{Fix}$-values routine from the previous section.

The following Lemma establishes an interpolation property that we use to modify Fix-values.

Lemma 5.1 Suppose $R \subseteq A^{n}$ is a compact representation, $i \in[n], \mathbf{a} \in A^{i}, \mathbf{b} \in R$ satisfying $a_{j}=b_{j}$ for all $1 \leq j<i$, and $c_{i}=d\left(a_{i}, b_{i}\right)$. Suppose further that

1. For each $I \subseteq[i]$ with $|I|=\min (k-1, i)$ and $i \in I$ we have an element $\mathbf{c}^{I} \in R$ with $\operatorname{proj}_{I}\left(\mathbf{c}^{I}\right)=\operatorname{proj}_{I}(\mathbf{a})$.

2. If $c_{i} \neq a_{i}$, then $\left(i, a_{i}, c_{i}\right)$ is witnessed in $R$, say by $\mathbf{u}, \mathbf{v}$.

Then $\left\{\mathbf{t} \in\langle R\rangle_{f}: t_{j}=a_{j}\right.$ for $\left.1 \leq j \leq i\right\} \neq \emptyset$.

PROOF: For notational convenience, if $c_{i}=a_{i}$ then let $\mathbf{u}=\mathbf{v}=\mathbf{c}^{[k-2] \cup\{i\}}$. We claim that the algorithm Interpolate found in Figure 2 returns an element in $\left\{\mathbf{t} \in\langle R\rangle_{f}: t_{j}=a_{j}\right.$ for $\left.1 \leq j \leq i\right\}$. For $i \geq k$, we prove by induction on $j=0,1, \ldots, i-k+1$ that if $I \subseteq\{j+1, j+2, \ldots, i\}$ with $|I|=k-1$ and $i \in I$, then $c_{t}^{j, I}=a_{t}$ for all $t \in[j] \cup I$.

The base of the induction, when $j=0$, follows since $\operatorname{proj}_{I}\left(\mathbf{c}^{I}\right)=\operatorname{proj}_{I}(\mathbf{a})$ and $\mathbf{c}^{0, I}=\mathbf{c}^{I}$. Assume that $j>0$ and that the $\mathbf{c}^{j-1, L}$ have the desired property for all $L \subseteq$ $\{j, j+1, \ldots, i\}$ with $|L|=k-1$ and $i \in L$. Straightforward calculations show that the elements $\mathbf{d}$ and $\mathbf{c}^{j, I}$ produced in Steps 3.1.3 and 3.1.5 of Interpolate are such that $d_{l}=$ $a_{l}$ for $l \in[j] \cup(I \backslash\{i\}), d_{i}=c_{i}$ and that $\operatorname{proj}_{[j] \cup I}\left(\mathbf{c}^{j, I}\right)=$ $\operatorname{proj}_{[j] \cup I}(\mathbf{a})$, as required.

We note that the run-time of Interpolate is $O\left(n^{k}\right)$ and so can be bounded by a polynomial in $n$.

Using the Interpolate algorithm we define the algorithm New-Fix-values as in Figure 3. Using New-Fix-values, we can define a relation (concept) from a given compact representation as follows:

Let $R$ be a compact representation of some $n$-ary relation over $A$. The concept encoded by $R$ is defined to be the set of all $\left(a_{1}, \ldots, a_{n}\right) \in A^{n}$ such that New-Fix-values $\left(R, a_{1}, \ldots, a_{n}\right)$ returns a non-empty set.

While the output of New-Fix-values may be hard to pin down when its input is a compact representation of no relation that is invariant under $f$, we can nevertheless state some useful properties of it.

Proposition 5.2 Let $R^{\prime}$ be a compact representation of some $n$-ary relation $R$ over $A$ and let $\mathbf{a}=\left(a_{1}, \ldots, a_{n}\right) \in$ $A^{n}$.

\section{The concept encoded by $R^{\prime}$ is contained in $\left\langle R^{\prime}\right\rangle_{f}$.}

2. If $R$ is invariant under $f$ then $\mathbf{a} \in R$ if and only New-Fix-values $\left(R^{\prime}, a_{1}, \ldots, a_{n}\right)$ is nonempty. Thus, if $R$ is invariant under $f$ then the concept encoded by $R^{\prime}$ is equal to $R$.

PROOF: A careful inspection of the definitions of Fix-values and New-Fix-values should convince the reader of the correctness of statement 1 . If $R$ is invariant under $f$ then by design, Fix-values $\left(R^{\prime}, a_{1}, \ldots, a_{n}\right)$ (and hence New-Fix-values $\left(R^{\prime}, a_{1}, \ldots, a_{n}\right)$ ) returns a compact representation of the subuniverse of $R$ that consists of all elements $\mathbf{b}$ of $R$ with $b_{i}=a_{i}$ for $1 \leq i \leq n$. Of course, this subuniverse is either empty (iff $\mathbf{a} \notin R$ ) or $\{\mathbf{a}\}$ and so $\mathbf{a} \in R$ if and only if New-Fix-values returns a non-empty set (which must be equal to $\{\mathbf{a}\}$ ). So, if $R$ is invariant under $f$ then the concept encoded by $R^{\prime}$ is equal to $R$.

Theorem 5.3 The concept class of relations over A, encoded by compact representations, is polynomially evaluable.

PROOF: Let $R \subseteq A^{n}$ be a compact representation. Since the run-times of $\mathrm{Fix}$-values and Interpolate can 


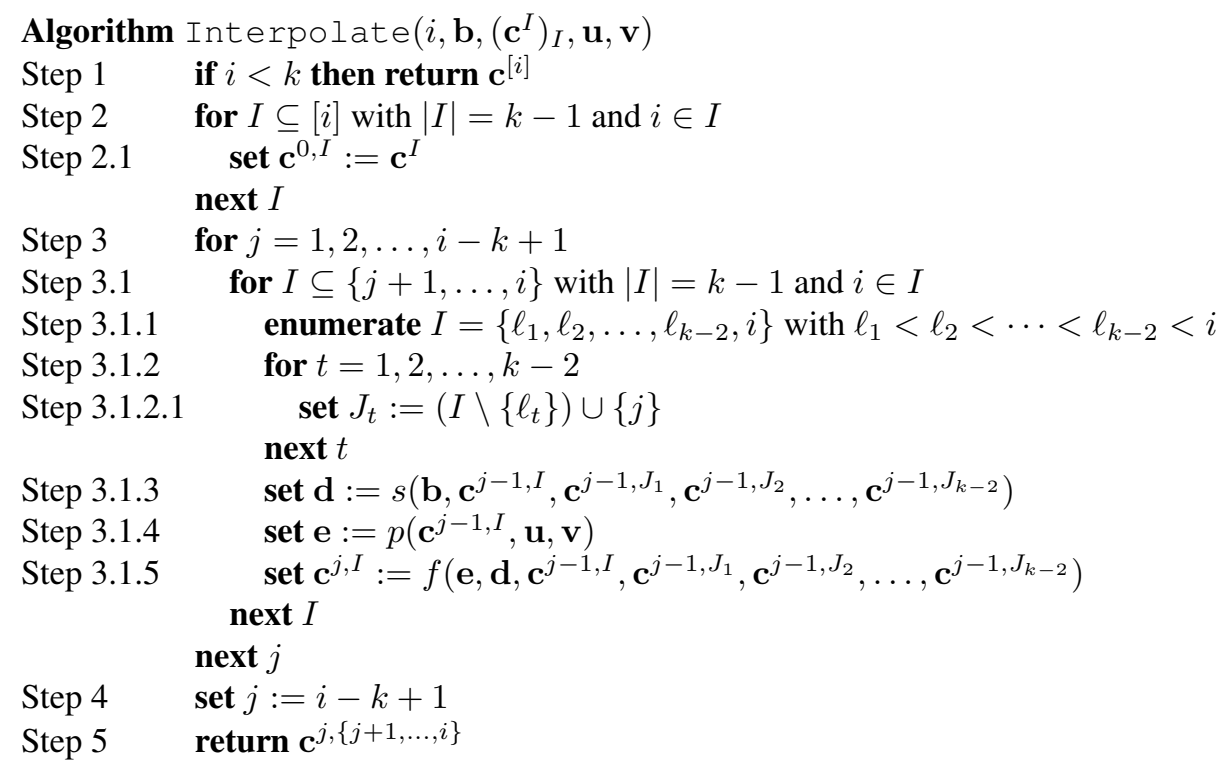

Figure 2. The Interpolate Algorithm

be bounded by some polynomial in $n$ it is not hard to see that the run-time of New-Fix-values $\left(R, a_{1}, \ldots, a_{n}\right)$ can also be bounded by a polynomial in $n$, for any $\mathbf{a} \in A^{n}$. From this it follows that membership in the relation encoded by $R$ can be determined in time bounded by a polynomial in $n$.

In Figure 4 we present a learning algorithm for the concept class of all finitary relations of $A$ invariant under $f$, coded by compact representations. We note that this algorithm is essentially the same as that found in [5]. In Step 2 of the algorithm, $E Q\left(R^{\prime}\right)$ represents the call to the oracle with hypothesis $R^{\prime}$. If $R^{\prime}$ does not code the target concept then $E Q$ returns a tuple from the symmetric difference of the target concept and the concept encoded by $R^{\prime}$. It can be shown inductively that $R^{\prime}$ is always a subset of the target concept and so $E Q$ will always return a tuple that lies in the target concept. Since in general, compact representations do not code relations invariant under $f$, this algorithm makes improper equivalence queries. We have:

Theorem 5.4 Let A be a finite set and $f$ a $k$-edge operation on $A$. Then there exists an algorithm that exactly learns $\operatorname{Inv}(f)$, encoded by compact representations, with improper equivalence queries and whose run-time is bounded by a polynomial in the arity of the target relation.

PROOF: Let $n$ be the arity of the target concept $T$. We will argue by induction that at any point in the execution of the learning algorithm the set $R^{\prime}$ is a compact representation of some $n$-ary relation contained in $T$ and that for each pass through the while-loop, the size of $R^{\prime}$ increases by one or two. Since the size of any compact representation of an $n$ ary relation over $A$ can be bounded by a polynomial in $n$, it follows that the run-time of the learning algorithm can be bounded by a polynomial in the arity of the target concept and that the output of the algorithm will encode the target concept.

Initially, $R^{\prime}=\emptyset$, a compact representation of the empty relation. Assume that at some later stage of the execution of the learning algorithm we have that $R^{\prime}$ is a compact representation. In Step 2, if the oracle returns the answer "yes" then the algorithm has successfully learned a code for the target concept. If, instead, the oracle returns an $n$-tuple $\mathbf{a}=\left(a_{1}, \ldots, a_{n}\right)$ then $\mathbf{a} \in T$ (but not in the relation coded by $R^{\prime}$ ) and $R^{\prime}$ will be increased in one of two ways.

If some projection of a onto a subset $I$ of coordinates of size $<k$ is not contained in $\operatorname{proj}_{I}\left(R^{\prime}\right)$ then a is added to $R^{\prime}$ and control passes again to Step 2. The new $R^{\prime}$ is still a compact representation since we have simply added an $n$-tuple to $R^{\prime}$ that witnesses that $\operatorname{proj}_{I}(\mathbf{a}) \in \operatorname{proj}_{I}(T)$.

If on the other hand, all projections of a onto small sets of coordinates are witnessed by members of $R^{\prime}$ then control passes to Step 2.2 and then we compute the smallest $i \leq n$ such that New-Fix-values $\left(R^{\prime}, a_{1}, \ldots, a_{i}\right)$ returns the empty set. Such an $i$ exists and $i>0$ since New-Fix-values $\left(R^{\prime}, a_{1}, \ldots, a_{n}\right)$ returns the empty set and New-Fix-values $\left(R^{\prime}\right)$ returns $R^{\prime}$.

Since New-Fix-values $\left(R^{\prime}, a_{1}, \ldots, a_{i}\right)$ returns the empty set then one of the conditions in the if statement in Step 4 of the execution of 


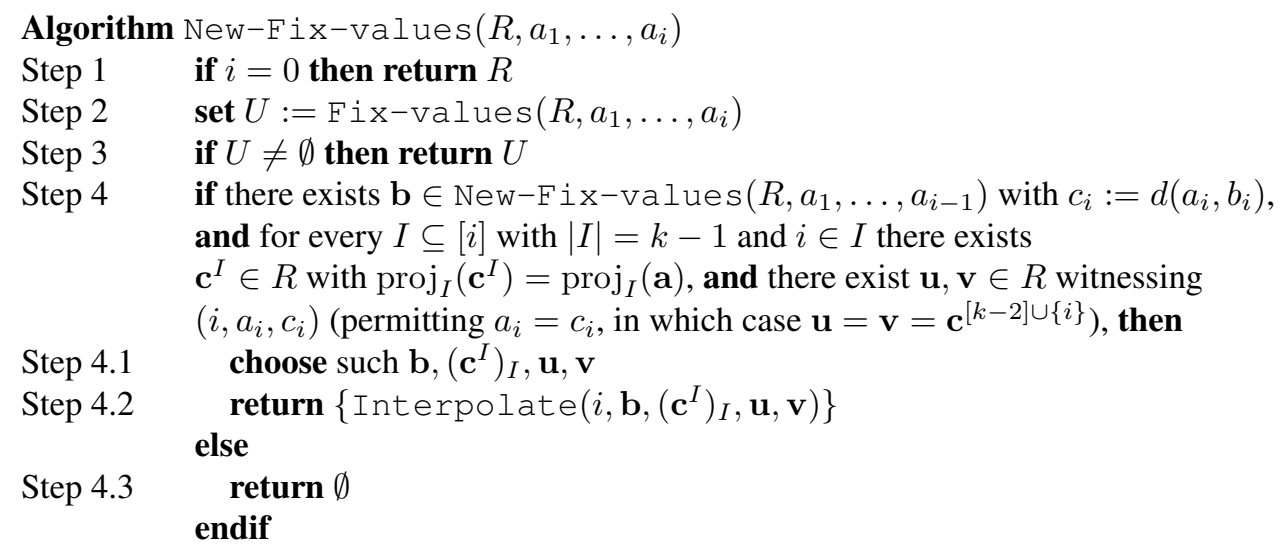

Figure 3. The New-Fix-values Algorithm

Step $1 \quad$ set $R^{\prime}:=\emptyset$

Step 2 while $E Q\left(R^{\prime}\right)=$ "no" do

let $\mathbf{a}=\left(a_{1}, \ldots, a_{n}\right)$ be the counterexample returned by $E Q$

Step 2.1 if there is some $k^{\prime}<k$ and $I=\left\{i_{1}, \ldots, i_{k^{\prime}}\right\} \subseteq[n]$ with $\operatorname{proj}_{I}(\mathbf{a}) \notin \operatorname{proj}_{I}\left(R^{\prime}\right)$ then

Step 2.1.1 set $R^{\prime}:=R^{\prime} \cup\{\mathbf{a}\}$

Step 2.2 else

Step 2.2.1 compute the least $i$ such that New-Fix-values $\left(R^{\prime}, a_{1}, \ldots, a_{i}\right)$ returns the empty set

Step 2.2.2 select $\mathbf{b}=\left(a_{1}, \ldots, a_{i-1}, b_{i}, \ldots, b_{n}\right)$ from the set returned by New-Fix-values $\left(R^{\prime}, a_{1}, \ldots, a_{i-1}\right)$

Step 2.2.3 set $R^{\prime}:=R^{\prime} \cup\{\mathbf{a}, d(\mathbf{a}, \mathbf{b})\}$

endif

endwhile

Step 3 return $R^{\prime}$

Figure 4. The Learning Algorithm

New-Fix-values $\left(R^{\prime}, a_{1}, \ldots, a_{i}\right)$ must fail. Note that since we are executing Step 2.2 of the learning algorithm at this point, it must be the case that for every $I \subseteq[i]$ with $|I|=k-1$ and $i \in I$ there exists $\mathbf{c}^{I} \in R$ with $\operatorname{proj}_{I}\left(\mathbf{c}^{I}\right)=\operatorname{proj}_{I}(\mathbf{a})$. By choice of $i$ we know that $\mathrm{New}-\mathrm{Fix}$-values $\left(R^{\prime}, a_{1}, \ldots, a_{i-1}\right)$ returns a non-empty set and for any $\mathbf{b}$ in this set, the element $c_{i}=d\left(a_{i}, b_{i}\right)$ is not equal to $a_{i}$ and the minority index $\left(i, a_{i}, c_{i}\right)$ is not witnessed in $R$. Thus for any selection of b in Step 2.2.2 of the learning algorithm we have that the minority index $\left(i, a_{i}, d\left(a_{i}, b_{i}\right)\right)$ is not witnessed in $R^{\prime}$ and hence, in Step 2.2.3, the set $R^{\prime} \cup\{\mathbf{a}, d(\mathbf{a}, \mathbf{b})\}$ is a compact representation that is strictly bigger than $R^{\prime}$ and that is contained in $T$.

Definition 5.5 Let $\Gamma$ be a set of finitary relations over the finite set $A$. The set of quantified generalized formulas over the basis $\Gamma$, denoted by $\forall \exists-F o r m(\Gamma)$, is the smallest set of first-order formulas over the variables $\left\{x_{1}, x_{2}, \ldots\right\}$ that is closed under conjunction and universal and existential quantification over the $x_{i}$ and that contains, for each $R \in \Gamma$ and sequence of variables $\mathbf{y}$, the formula $R(\mathbf{y})$ (where the arity of $R=$ the length of $\mathbf{y}$ ).

Using the usual semantics of first-order logic, each member $\Phi$ of $\forall \exists$-Form $(\Gamma)$ defines a relation $R_{\Phi}$ on $A$. With this in mind, we also use $\forall \exists$-Form $(\Gamma)$ to denote the concept class of all relations of the form $R_{\Phi}$, for $\Phi \in \forall \exists$-Form $(\Gamma)$, coded by $\Phi$.

Corollary 5.6 Let $A$ be a set and $f$ a k-edge operation on $A$. If $\Gamma$ is a subset of $\operatorname{Inv}(f)$ then $\forall \exists$-Form $(\Gamma)$ is polynomially exactly learnable with improper equivalence queries.

PROOF: Lemma 1 from [5] establishes that $R_{\Phi}$ is a member of $\operatorname{Inv}(f)$ for any $\Phi \in \forall \exists-\operatorname{Form}(\Gamma)$. From this, the result follows from the previous theorem. 
We note that Theorem 5.4 and Corollary 5.6 extend results found in [13] and [5]. They also settle one direction of a conjecture found in [11] and [10].

\section{Conclusion}

The study of algebras with few subpowers has led to a number of surprising algebraic results and has helped to further work on the Dichotomy Conjecture for constraint languages. As noted earlier, our results delimit the scope of Dalmau-like algorithms for solving instances of the CSP and so new algorithms will need to be devised to deal with finite algebras that support non-trivial term operations more general than $k$-edge terms.

Given a finite algebra (or constraint language) there is a fairly straightforward, but inefficient algorithm to determine if it has a Mal'cev term operation (or Mal'cev polymorphism). In [15] a polynomial time algorithm to test for a Mal'cev term is presented that works for idempotent algebras. It is apparently much more difficult to test for the presence of a near unanimity term, but at least we know from a recent result of Maróti [18] that this question is decidable.

Question 1: Is there an algorithm to determine, given a finite algebra (or finite constraint language), whether it has a $k$-edge term operation (polymorphism) for some $k>1$ ?

A more direct approach to learnability would be to use generating sets as codes for subpowers of a finite algebra, rather than compact representations. Theorem 5.4 and Corollary 5.6 would have much cleaner proofs if the following question has an affirmative answer.

Question 2: Let A be a finite algebra with few subpowers. Is there a polynomial time algorithm that takes as input a subset $R$ and an $n$-tuple a from $A^{n}$ for some $n$, and determines if $\mathbf{a}$ is in the subuniverse of $\mathbf{A}^{n}$ generated by $R$ ? An algebra with this property is said to be polynomially evaluable (see [13]).

\section{References}

[1] D. Angluin and M. Kharitonov. When won't membership queries help? J. Comput. System Sci., 50(2):336-355, 1995. 23rd Symposium on the Theory of Computing (New Orleans, LA, 1991).

[2] J. Berman, P. Idziak, P. Markovic, R. McKenzie, M. Valeriote, and R. Willard. Varieties with few subalgebras of powers. preprint, 2006.

[3] A. Bulatov. Tractable conservative constraint satisfaction problems. In P. G. Kolaitis, editor, Proceedings of the Eighteenth Annual IEEE Symp. on Logic in Computer Science, LICS 2003, pages 321-330. IEEE Computer Society Press, June 2003.
[4] A. Bulatov. A dichotomy theorem for constraint satisfaction problems on a 3-element set. J. ACM, 53(1):66-120 (electronic), 2006.

[5] A. Bulatov, H. Chen, and V. Dalmau. Learning intersectionclosed classes with signatures. to appear in Theoretical Computer Science.

[6] A. Bulatov and V. Dalmau. A simple algorithm for Mal'tsev constraints. SIAM J. Comput., 36(1):16-27 (electronic), 2006.

[7] A. Bulatov and P. Jeavons. Algebraic structures in combinatorial problems. submitted for publication.

[8] A. Bulatov, P. Jeavons, and A. Krokhin. Classifying the complexity of constraints using finite algebras. SIAM J. Comput., 34(3):720-742 (electronic), 2005.

[9] S. Burris and H. P. Sankappanavar. A course in universal algebra, volume 78 of Graduate Texts in Mathematics. Springer-Verlag, New York, 1981.

[10] H. Chen. The expressive rate of constraints. Ann. Math. Artif. Intell., 44(4):341-352, 2005.

[11] V. Dalmau. Computational complexity of problems over generalized formulas. PhD thesis, Universitat Politécnica de Catalunya, 2000.

[12] V. Dalmau. Generalized majority-minority operations are tractable. In P. Panangaden, editor, Proceedings of the Twentieth Annual IEEE Symp. on Logic in Computer Science, LICS 2005, pages 438-447. IEEE Computer Society Press, June 2005.

[13] V. Dalmau and P. Jeavons. Learnability of quantified formulas. Theoret. Comput. Sci., 306(1-3):485-511, 2003.

[14] T. Feder and M. Y. Vardi. The computational structure of monotone monadic SNP and constraint satisfaction: a study through Datalog and group theory. SIAM J. Comput., 28(1):57-104 (electronic), 1999.

[15] R. S. Freese and M. A. Valeriote. Mal'cev conditions and idempotent algebras. preprint, 2006.

[16] P. Jeavons. On the algebraic structure of combinatorial problems. Theoret. Comput. Sci., 200(1-2):185-204, 1998.

[17] P. Jeavons, D. Cohen, and M. C. Cooper. Constraints, consistency and closure. Artificial Intelligence, 101(1-2):251265, 1998.

[18] M. Maróti. The existence of a near-unanimity term in a finite algebra is decidable. preprint, 2005.

[19] R. McKenzie, G. McNulty, and W. Taylor. Algebras, Lattices, Varieties Volume 1. Wadsworth and Brooks/Cole, Monterey, California, 1987.

[20] T. J. Schaefer. The complexity of satisfiability problems. In Conference Record of the Tenth Annual ACM Symposium on Theory of Computing (San Diego, Calif., 1978), pages 216226. ACM, New York, 1978. 